\title{
Heart Myofibrillogenesis Occurs in Isolated Chick Posterior Blastoderm: A Culture Model
}

\author{
Hiroko Matsui ${ }^{1}$, Masahide Sakabe ${ }^{1}$, Hirokazu Sakata ${ }^{1}$, Kazuki Nakatani ${ }^{1}$, Kazuo Ikeda ${ }^{1}$, \\ Mitsuru Fukui ${ }^{2}$, Katsumi Ando ${ }^{3}$, Toshiyuki Yamagishi ${ }^{3}$ and Yuji Nakajima ${ }^{1}$
}

\author{
${ }^{1}$ Department of Anatomy and Cell Biology and ${ }^{2}$ Laboratory of Statistics, Graduate School of Medicine, Osaka City University, \\ 1-4-3 Asahimachi, Abenoku, Osaka 545-8585, Japan and ${ }^{3}$ Department of Anatomy, School of Medicine, Saitama Medical \\ University, 38 Morohongo, Moroyama-cho, Irumagun, Saitama 350-0495, Japan
}

Received April 6, 2006; accepted September 21, 2006; published online October 11, 2006

\begin{abstract}
Early cardiogenesis including myofibrillogenesis is a critical event during development. Recently we showed that prospective cardiomyocytes reside in the posterior lateral blastoderm in the chick embryo. Here we cultured the posterior region of the chick blastoderm in serum-free medium and observed the process of myofibrillogenesis by immunohistochemistry. After 48 hours, explants expressed sarcomeric proteins (sarcomeric $\alpha$-actinin, 61\%; smooth muscle $\alpha$-actin, 95\%; Z-line titin, 56\%; sarcomeric myosin, 48\%); however, they did not yet show a mature striation. After 72 hours, more than $92 \%$ of explants expressed I-Z-I proteins, which were incorporated into the striation in $75 \%$ of explants or more (sarcomeric $\alpha$-actinin, $75 \%$; smooth muscle $\alpha$-actin, $81 \%$; Z-line titin, 83\%). Sarcomeric myosin was expressed in $63 \%$ of explants and incorporated into A-bands in $37 \%$. The percentage incidence of expression or striation of I-Z-I proteins was significantly higher than that of sarcomeric myosin. Results suggested that the nascent I-Z-I components appeared to be generated independently of A-bands in the cultured posterior blastoderm, and that the process of myofibrillogenesis observed in our culture model faithfully reflected that in vivo. Our blastoderm culture model appeared to be useful to investigate the mechanisms regulating the early cardiogenesis.
\end{abstract}

Key words: myofibrillogenesis, early cardiogenesis, blastoderm, chick embryo

\section{Introduction}

In early chick cardiogenesis, prospective heart cells are located in posterior lateral region of the epiblast, but not in central to anterior epiblast $[5,14,23]$. During gastrulation, the prospective heart cells migrate into the anterior half of the primitive streak, subsequently leave the primitive streak and accumulate in the anterior region of the lateral plate mesoderm, called the precardiac mesoderm [11]. The anterior lateral plate mesoderm then divides dorsoventrally into the somatic and the visceral mesoderm. The left and right visceral mesoderm migrate anterior midline of the intestinal

Correspondence to: Yuji Nakajima M.D., Department of Anatomy and Cell Biology, Graduate School of Medicine, Osaka City University, 1-4-3 Asahimachi, Abenoku, Osaka 545-8585, Japan.

E-mail: yuji@med.osaka-cu.ac.jp portal and fuse to form the primitive heart tube. At this stage (late gastrula to neurula, Hamburger and Hamilton [HH]stage 6-10) [13], myofibrillogenesis occurs rapidly and the heart starts to beat spontaneously [15].

The processes of de novo myofibrillogenesis are highly conserved in vertebrate striated muscle including nascent cardiomyocytes, and so investigators have tried to explain the process involved in the formation of the sarcomeric structure, which consists of an organized I-Z-I component and A-band [26]. Several models have been proposed to explain how myofibrillogenesis is carried out during cardiac muscle differentiation using neonatal/fetal cardiomyocyte culture systems, in which isolated cardiomyocytes lose their mature myofibrils and reorganize myofibrils during cultivation. It has been reported that the first step involves a stressfiber-like structure as a scaffold, then the I-Z-I proteins (the Z-disk components being sarcomeric $\alpha$-actinin, $\alpha$-actin 
and Z-line titin) and thick A-bands (consisting mainly of myosin) are assembled independently to later become incorporated into myofibrils [16]. At the onset of myofibrillogenesis, the stress-fiber-like structure consists of mini-sarcomeres containing actin filaments, $\alpha$-actinin and non-muscle myosin. Subsequently, the distance between the dense materials of the nascent myofibril increases, titin is incorporated, and non-muscle myosin is replaced by musclespecific myosin to complete the mature myofibrils $[19,30]$. In vivo observations of myofibrillogenesis in chick embryonic heart showed that the myofibrillogenesis occurs in a similar manner to that observed in culture models [8].

Despite many studies, there is still uncertainty about the morphological and molecular mechanisms that are required for early cardiogenesis, such as heart mesoderm formation, heart specification and terminal differentiation. To establish a culture system for investigation of the early cardiomyogenesis, we cultured chick posterior blastoderm in serum-free defined medium and observed the process of myofibrillogenesis by immunohistochemistry.

\section{Materials and Methods}

\section{Culture procedures}

Eyal-Giladi and Kochav (EK)-stage X-XI blastoderms (incubation time $0 \mathrm{hr}$ ) [10] were collected on ice-cooled phosphate-buffered saline (PBS), and the posterior blastoderm including the epiblast and associated hypoblast, but not sickle, was isolated using a sharp tungsten needle (hatched square in Fig. 1). The resulting explants were explanted onto chamber slides (Nunc) and cultured in a serum-free defined medium (75\% DMEM, 25\% McCoy's medium, supplemented with $10^{-7} \mathrm{M}$ dexamethasone and penicillin-streptomycin, Sigma, St. Louis, MO, USA) [18].

\section{Indirect immunofluorescence microscopy}

Immunohistochemistry was performed, as described elsewhere [24]. Cultures were drained of medium, fixed with $4 \%$ paraformaldehyde/PBS for $30 \mathrm{~min}$ at room temperature, and rinsed with PBS. Specimens were blocked for $1 \mathrm{hr}$ with $1 \%$ BSA (bovine serum albumin) containing $0.1 \%$ Triton $\mathrm{X}-100$, and then incubated with primary antibody mixture at $4^{\circ} \mathrm{C}$ overnight. They were then rinsed with PBS and incubated with FITC- or RITC-conjugated secondary antibody mixture for $1 \mathrm{hr}$ at room temperature. Samples were observed under a laser confocal microscope (Zeiss). Explants that expressed sarcomeric proteins or generated mature striations were counted under a microscope. Percentage incidence of sarcomeric protein- or striation-positive explants was calculated. Statistical analyses were performed using Fisher's exact test and Bonferroni's correction for multiple comparison. Significance level was less than 5\%.

\section{Antibodies}

The monoclonal antibodies anti-Z-line titin (anti-titin, clone 9D10, IgM, supernatant), anti-sarcomeric myosin heavy chain (clone MF20, IgG2b, supernatant) [3] and anti-

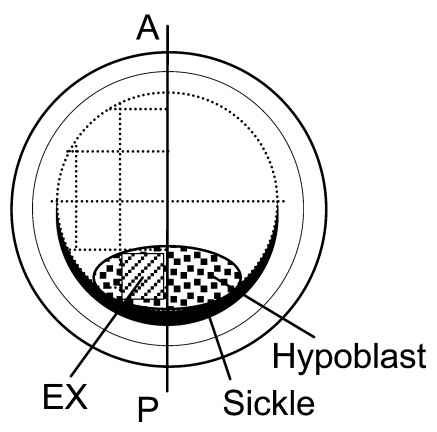

\section{Stage XI blastoderm}

(ventral view)

Fig. 1. Explant from prestreak chick blastoderm. EK-stage $\mathrm{X}-\mathrm{XI}$ blastoderms were collected. Posterior regions (hatched square) containing epiblast and hypoblast were isolated and cultured in serumfree medium. Note that the EK-stage indicates the embryonic stages before gastrulation by Eyal-Giladi and Kochav (1976) [10]. A, anterior; $\mathrm{P}$, posterior.

skeletal muscle sarcoplasmic reticulum (clone 12/101, IgG1, supernatant) [12] were obtained from the Developmental Studies Hybridoma Bank (Iowa City, IA, USA). The monoclonal antibodies, anti-smooth muscle $\alpha$-actin (SMA, clone 1A4, IgG2a, 1:400) [28] and anti-sarcomeric $\alpha$-actinin (clone EA53, IgG1, 1:600), were purchased from Sigma (St. Louis, MO, USA). Anti-Nkx2.5 (goat IgG, 1:100) was purchased from Santa Cruz Biotechnology (Santa Cruz, Ca, USA). For double immunohistochemistry, we used FITCconjugated goat anti-mouse IgG2a, RITC-conjugated goat anti-mouse IgG2a, FITC-conjugated goat anti-mouse IgG1, FITC-conjugated goat anti-mouse IgG2b, RITC-conjugated goat anti-mouse IgM (Southern Biotechnology, Birmingham, AL, USA) and FITC-conjugated donkey anti-goat IgG (Chemicon, Temecula, CA, USA) as secondary antibodies. Secondary antibodies we used were diluted 1:100 and did not cross-react with chick embryonic tissue (data not shown).

\section{Results}

Prospective heart-forming posterior blastoderm from EK-stage X-XI embryos (hatched region in Fig. 1) was cultured in serum-free defined medium, fixed and doubly stained with antibodies against various cardiomyocytespecific proteins, such as Nkx-2.5, smooth muscle $\alpha$-actin (SMA), sarcomeric $\alpha$-actinin, Z-line titin and sarcomeric myosin. It has been reported that SMA is initially expressed in precardiac mesoderm at HH-stage 6 (late gastrula) and then recruited as an initial heart $\alpha$-actin $[6,29]$. After $24 \mathrm{hr}$ in culture, there was no detectable staining for these heartspecific proteins (data not shown). After $48 \mathrm{hr}$ in culture, the heart-specific transcription factor Nkx-2.5 was detectable in the nuclei of cells that were expressing sarcomeric $\alpha$-actinin (Fig. 2) [17]. At this time, tiny bead-like deposits of sarcomeric $\alpha$-actinin were observed $(61 \%$ of explants, $n=28)$ 

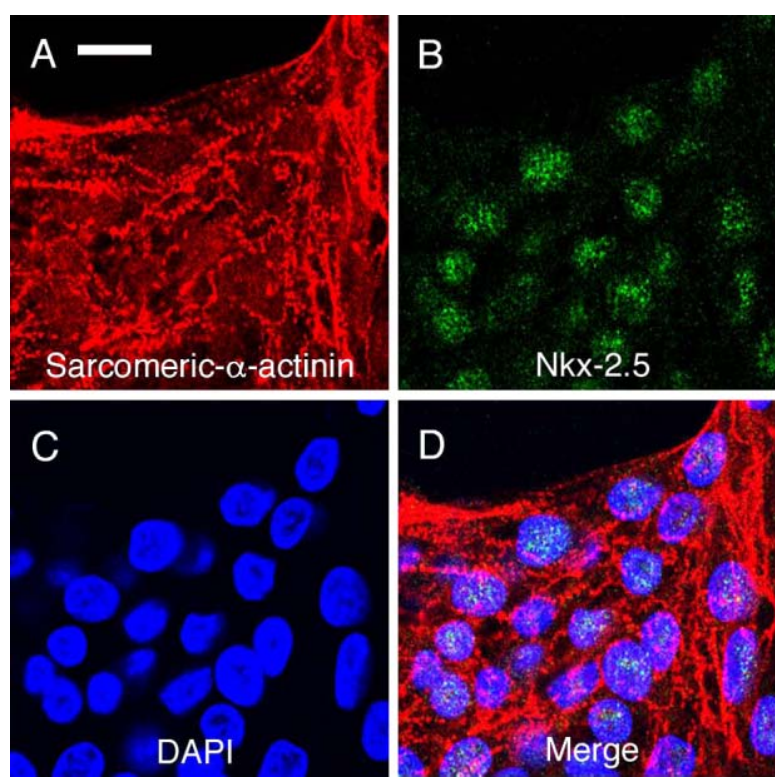

Fig. 2. Cultured posterior blastoderm expresses the heart-specific transcription factor $\mathrm{Nkx}-2.5$. After $48 \mathrm{hr}$ in culture, explants were fixed and stained with antibodies against heart specific transcription factor $(\mathrm{Nkx}-2.5)$ and early heart sarcomeric protein (sarcomeric $\alpha$-actinin). Cells that were expressing sarcomeric $\alpha$-actinin (A) expressed Nkx-2.5 (B) in their nuclei $(\mathbf{C}, \mathbf{D})$. Bar $=20 \mu \mathrm{m}$. along the SMA-positive $(95 \%, \mathrm{n}=19)$ filaments, which were terminating at epithelial-cell borders (Figs. 3A, 5). Sarcomeric $\alpha$-actinin and SMA were also present around the circumference at the cell-cell border. At this time, no apparent mature sarcomeric structure consisting of sarcomeric $\alpha$-actinin and SMA was observed (Fig. 5). The amino terminal domain of titin (Z-line titin) was expressed (56\%, $\mathrm{n}=18$ ) and co-localized with the bead-like deposit of sarcomeric $\alpha$-actinin (Figs. 3B, 5). Sarcomeric myosin was distributed in a diffuse manner $(46 \%, \mathrm{n}=28)$ in cells within which bead-like sarcomeric $\alpha$-actinin was observed (Figs. $3 \mathrm{C}, 5$ ). After $72 \mathrm{hr}$ in culture, $92 \%$ or more of explants expressed sarcomeric $\alpha$-actinin $(92 \%, \mathrm{n}=64)$, SMA $(100 \%$, $\mathrm{n}=37)$ and Z-line titin (94\%, $\mathrm{n}=36)$. Sarcomeric myosin was expressed in $63 \%$ of explants $(n=49)$. At this time, $75 \%$ or more of explants exhibited sarcomeric staining of sarcomeric $\alpha$-actinin (75\%), SMA (81\%) and Z-line titin (83\%). By contrast, A-band associated sarcomeric myosin was exhibited in 37\% of explants (Figs. 4, 5). Statistical analysis (Fisher's exact test) showed that there was significant difference in the percentage incidence of expression or striation among four sarcomeric proteins such as sarcomeric $\alpha$-actinin, SMA, Z-line titin and myosin. The results of each individual pairwise comparison are indicated in Figure 5. There was no significant difference in the incidence of expression or striation among I-Z-I proteins, and the incidence of expres-
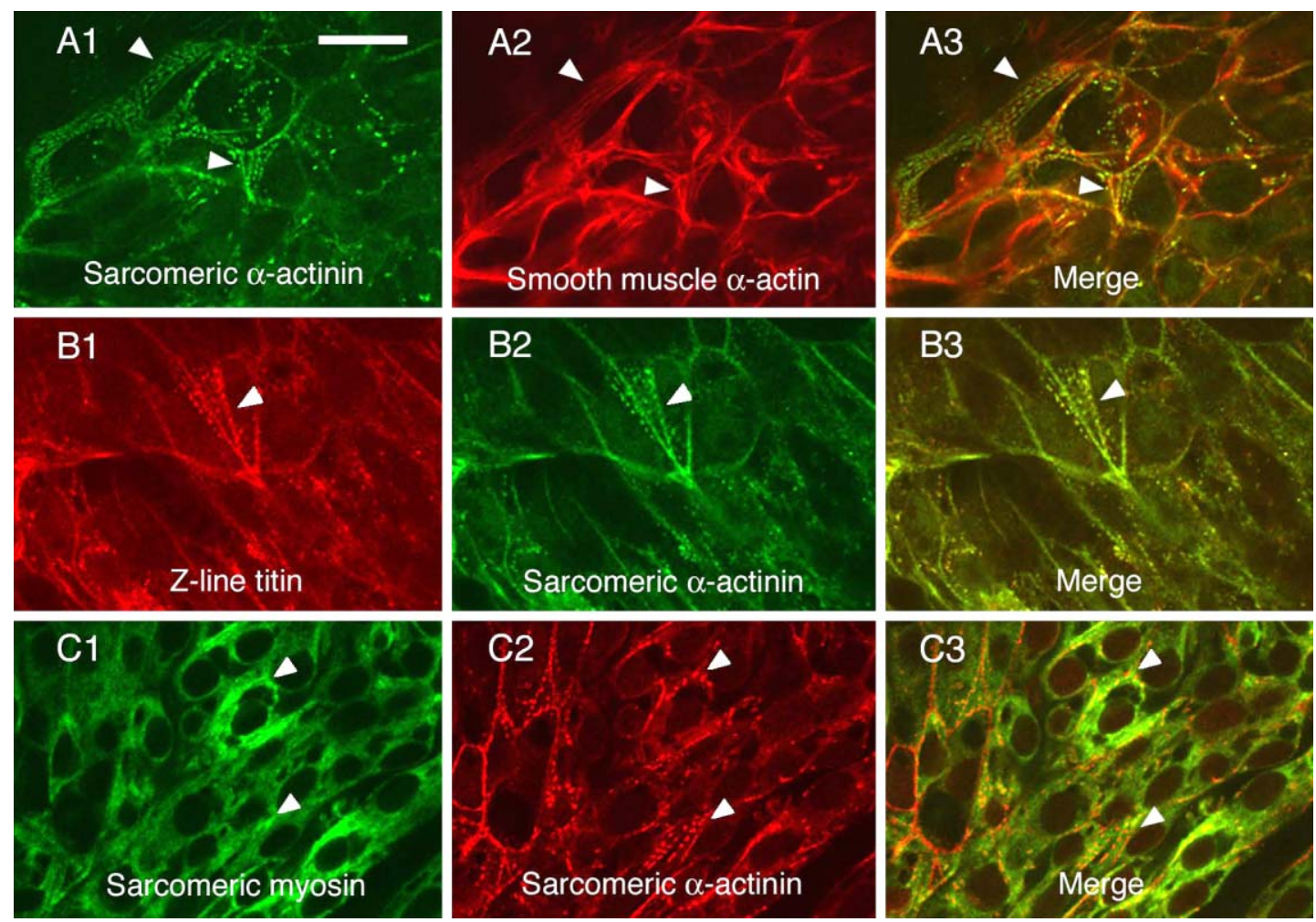

Fig. 3. EK-stage X-XI posterior blastoderm generates premature myofibrils after $48 \mathrm{hr}$ in culture. Tiny bead-like deposits of sarcomeric $\alpha$ actinin (arrowheads in A1) appeared along filamentous smooth muscle $\alpha$-actin (arrowheads in A2-3). Z-line titin was also seen as tiny bead-like deposits and co-localized with the sarcomeric $\alpha$-actinin (arrowheads in B1-3). Sarcomeric myosin exhibited a diffuse cytoplasmic staining (C1) and was distributed independently of the bead-like deposits of sarcomeric $\alpha$-actinin (arrowheads in C2 and C3). Bar=20 $\mu \mathrm{m}$. 

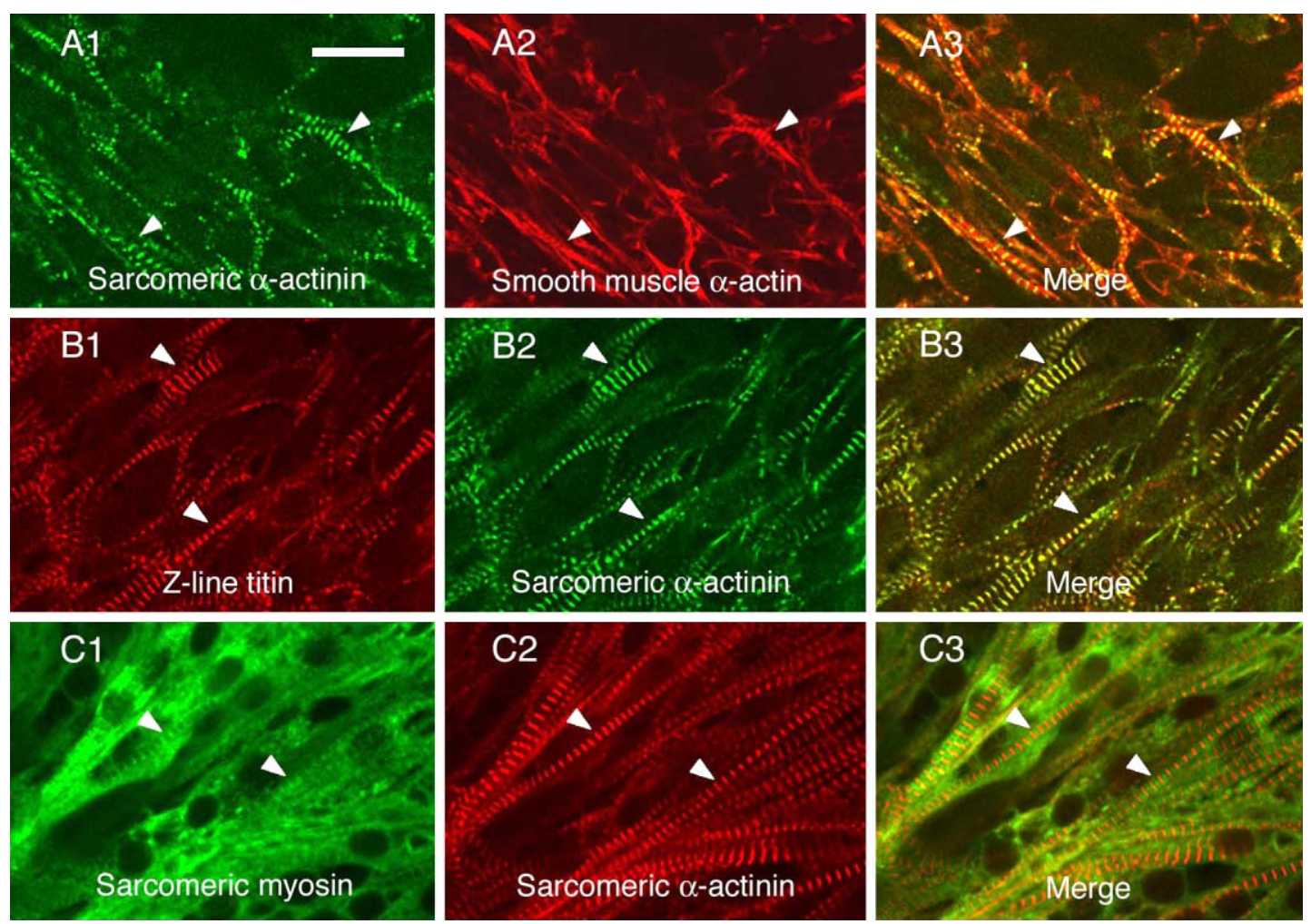

Fig. 4. EK-stage $\mathrm{X}$-XI posterior blastoderm generates mature myofibrils after $72 \mathrm{hr}$ in culture. Sarcomeric $\alpha$-actinin (A1) and smooth muscle $\alpha$ actin (A2) were incorporated into mature I-Z-I structures (arrowheads in A1-3). Z-line titin was co-localized with striated sarcomeric $\alpha$-actinin (arrowheads in B1-3). Sarcomeric myosin was also incorporated into nascent sarcomeres (arrowheads in $\mathbf{C 1}$ ) and associated with sarcomeric $\alpha$ actinin (arrowheads in C2-3). Bar=20 $\mu \mathrm{m}$.
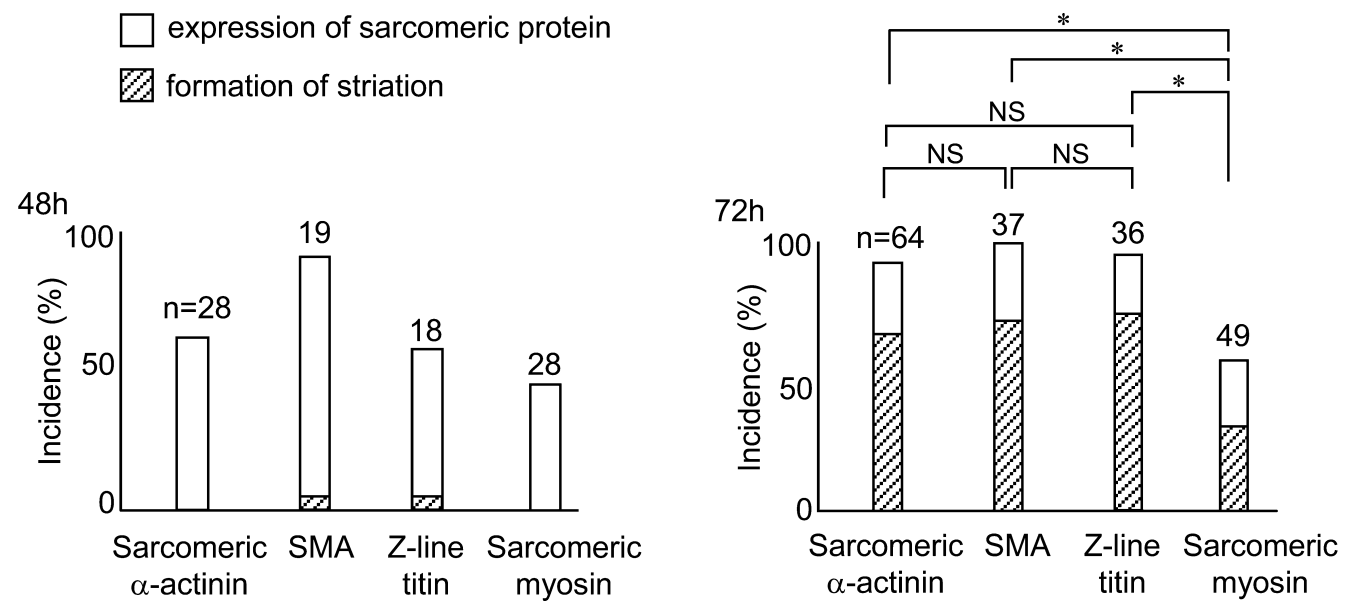

Fig. 5. Percentage incidence of the expression of sarcomeric proteins and striation in cultured posterior blastoderm shown in Figures 3 and 4. After $48 \mathrm{hr}(\mathrm{h})$ in culture, explants expressed sarcomeric proteins (sarcomeric $\alpha$-actinin, $61 \%$; smooth muscle $\alpha$-actin (SMA), $95 \%$; Z-line titin, $56 \%$; sarcomeric myosin, 48\%); however, they did not yet show an apparent striation (hatched column). After $72 \mathrm{hr}$, explants expressed sarcomeric proteins (sarcomeric $\alpha$-actinin, 92\%; SMA, 100\%; Z-line titin, 94\%; sarcomeric myosin, 63\%). I-Z-I proteins were incorporated into the striation in $75 \%$ of explants or more (sarcomeric $\alpha$-actinin, 75\%; SMA, 81\%; Z-line titin, 83\%), and sarcomeric myosin in 37\% of explants. There was a significant difference in the percentage incidence of expression or striation among four sarcomeric proteins. The results of each individual pairwise comparison were indicated. Note that there was no significant difference in the incidence of expression or striation of I-Z-I proteins and that the incidence of expression or striation of I-Z-I proteins was significantly higher than that of sarcomeric myosin. Asterisk $(*)$ indicates that there is $5 \%$ significant difference $(\mathrm{P}<0.05 / 6$, using Bonferroni's correction). NS, no significant difference; $\mathrm{n}$, number of explants tested; SMA, smooth muscle $\alpha$-actin. 
sion or striation of I-Z-I proteins was significantly higher than that of sarcomeric myosin.

It has been reported that the posterior blastoderm contains not only prospective heart cells but also skeletal muscle [14]. Therefore, we checked the expression of a skeletal muscle marker, 12/101, in cultured posterior blastoderm. However, there was no detectable immunoreactivity to the anti-skeletal sarcoplasmic reticulum antibody (12/101 antibody, data not shown), indicating that mature skeletal muscle had yet to develop after $72 \mathrm{hr}$ in culture. This may be because mature skeletal muscle does not develop in ovo during $72 \mathrm{hr}$ of incubation [1].

\section{Discussion}

In the present paper, we described that cultured posterior blastoderm (epiblast cells associated with subjacent hypoblast cells) in serum-free defined medium was able to generate mature myofibrils with sarcomeres. At the onset of myofibrillogenesis (after $48 \mathrm{hr}$ in culture), actin filaments of SMA were associated with periodic tiny bead-like deposits of sarcomeric $\alpha$-actinin and Z-line titin; at this time, the deposition of sarcomeric myosin appeared to be diffuse. After $72 \mathrm{hr}$, although $75 \%$ or more of explants showed sarcomeric staining of sarcomeric $\alpha$-actinin, SMA and Z-line titin, sarcomeric myosin was incorporated into the sarcomere in only $37 \%$ of explants. Therefore, in this culture model, myofibrillogenesis took place after $48 \mathrm{hr}$ in culture and mature myofibrils with sarcomeres developed by $72 \mathrm{hr}$. Our immunohistochemical observation for myofibrillogenesis supported the schema that the nascent I-Z-I components consisting of SMA, sarcomeric $\alpha$-actinin, and Z-line titin might be generated independently of sarcomeric myosin-associated A-bands, which would be recruited into the I-Z-I structure to generate mature myofibrils $[16,19,30]$. Therefore, the developmental process of the myofibrillogenesis observed in the cultured posterior blastoderm mimicked that in vivo [8], in cultured cardiomyocytes [16, 19] and in cultured anterior lateral plate mesoderm (heart mesoderm) [7, 24, 25].

The earliest development of the heart at gastrula stage has been investigated extensively using avian embryos, as they are easy to handle in vivo and in vitro. During gastrulation, presumptive heart cells are committed to the cardiac lineage and terminal differentiation occurs at around the gastrula to neurula stages (for reviews [4, 21]). This specification and the subsequent terminal differentiation processes are regulated by endoderm-derived bone morphogenetic protein (BMP)-2/4, fibroblast growth factor (FGF)8 and antagonists to the canonical Wnt-pathway [2, 9, 20, $22,24,27]$. In pregastrula embryos, the signaling regulating the formation of the heart-forming mesoderm as well as cardiomyocytes is not yet well understood. Explantation experiments using an avian blastoderm culture showed that the posterior epiblast develops into heart mesoderm as well as cardiomyocytes, if cultured with associated hypoblast or medium supplemented with activin $[18,23,31]$. There- fore, tissue interaction between the posterior epiblast and hypoblast is required to generate heart mesoderm/cardiomyocytes $[23,31]$. However, the molecular mechanisms that regulate the induction of heart mesoderm/cardiomyocytes from the posterior epiblast remain uncertain. To investigate the molecular mechanisms regulating early cardiogenesis, our serum-free culture model would be convenient, as the histogenesis in vitro faithfully reflects that in vivo and lossof-function and gain-of-function experiments are easy to perform.

\section{Acknowledgments}

This work was supported by Grants-in-Aid from the Ministry of Education, Culture, Sports, Science, and Technology of Japan (\#17590169 and 17-3456), the Japan Cardiovascular Research Foundation, the Takeda Science Foundation, the Terumo Life Science Foundation, and the Miyata Heart Foundation. Some of the monoclonal antibodies used were obtained from the Developmental Studies Hybridoma Bank (University of Iowa, Iowa City, IA, USA).

\section{References}

1. Allen, E. R. and Pepe, F. A. (1965) Ultrastructure of developing muscle cells in the chick embryo. Am. J. Anat. 116;115-147.

2. Alsan, B. H. and Schultheiss, T. M. (2002) Regulation of avian cardiogenesis by Fgf8 signaling. Development 129; 1935-1943.

3. Bader, D., Masaki, T. and Fischman, D. A. (1982) Immunochemical analysis of myosin heavy chain during avian myogenesis in vivo and in vitro. J. Cell Biol. 95; 763-770.

4. Brand, T. (2003) Heart development: molecular insight into cardiac specification and early morphogenesis. Dev. Biol. 258; 1-19.

5. Callebaut, M. (2005) Origin, fate, and function of the components of the avian germ disc region and early blastoderm: role of ooplasmic determinants. Dev. Dyn. 233; 1194-1216.

6. Colas, J.-F., Lawson, A. and Schoenwolf, G. C. (2000) Evidence that transition of smooth muscle alpha-actin mRNA is delayed in the chick promyocardium until fusion of bilateral heart-forming regions. Dev. Dyn. 218; 316-330.

7. Du, A., Sanger, J. M., Linask, K. K. and Sanger, J. W. (2003) Myofibrillogenesis in the first cardiomyocyte formed from isolated quail precardiac mesoderm. Dev. Biol. 257; 382-394.

8. Ehler, E., Rothen, B. M., Hammerle, S. P., Komiyama, M. and Perriard, J.-C. (1999) Myofibrillogenesis in the developing chicken heart: assembly of Z-disk, M-line and thick filaments. J. Cell Sci. 112; 1529-1539.

9. Eisenberg, C. A. and Eisenberg, L. M. (1999) WNT11 promotes cardiac tissue formation of early mesoderm. Dev. Dyn. 216; 4558.

10. Eyal-Giladi, H. and Kochav, S. (1976) From cleavage to primitive streak formation: a complementary normal table and a new look at the first stage of the development of the chick. I. General morphology. Dev. Biol. 49; 321-337.

11. Garcia-Martinez, V. and Schoenwolf, G. C. (1993) Primitivestreak origin of cardiovascular system in avian embryos. Dev. Biol. 159; 706-719.

12. Griffin, K. J., Fekete, D. M. and Carlson, B. M. (1987) A monoclonal antibody stains myogenic cells in regenerating newt muscle. Development 101; 267-277.

13. Hamburger, V. and Hamilton, H. L. (1992) A series of normal stages in the development of the chick embryo. Dev. Dyn. 195; $231-272$. 
14. Hatada, Y. and Stern, C. D. (1994) A fate map of the epiblast of the early chick embryo. Development $120 ; 2879-2889$.

15. Hiruma, T. and Hirakow, R. (1985) An ultrastructural topographical study on myofibrillogenesis in the heart of the chick embryo during pulsation onset period. Anat. Embryol. 172; 325-329.

16. Holtzer, H., Hijikata, T., Lin, Z. X., Zhang, Z. Q., Holtzer, S., Protasi, F., Franzini-Armstrong, C. and Sweeney, H. L. (1997) Independent assembly of $1.6 \mu \mathrm{m}$ long bipolar MHC filaments and I-Z-I bodies. Cell Struct. Funct. 22; 83-93.

17. Komuro, I. and Izumo, S. (1993) Csx: A murine homeoboxcontaining gene specifically expressed in the developing heart. Proc. Natl. Acad. Sci. US A 90; 8145-8149.

18. Ladd, A. N., Yatskievych, T. A. and Antin, P. B. (1998) Regulation of avian cardiac myogenesis by Activin/TGF $\beta$ and bone morphogenetic proteins. Dev. Biol. 204; 407-419.

19. LoRusso, S. M., Rhee, D., Sanger, J. M. and Sanger, J. W. (1997) Premyofibrils in spreading adult cardiomyocytes in tissue culture: evidence for reexpression of the embryonic program for myofibrillogenesis in adult cells. Cell Motil. Cytoskeleton 37; 183-198.

20. Lough, J., Barron, M., Brogley, M., Sugi, Y., Bolender, D. L. and Zhu, X. (1996) Combined BMP-2 and FGF-4, but neither factor alone, induces cardiogenesis in non-precardiac embryonic mesoderm. Dev. Biol. 178; 198-202.

21. Lough, J. and Sugi, Y. (2000) Endoderm and heart development. Dev. Dyn. 217; 327-342

22. Marvin, M. J., Di Rocco, G., Gardiner, A., Bush, S. M. and Lassar, A. B. (2001) Inhibition of Wnt activity induces heart formation from posterior mesoderm. Genes Dev. 15; 316-327.

23. Matsui, H., Ikeda, K., Nakatani, K., Sakabe, M., Yamagishi, T.,
Nakanishi, T. and Nakajima, Y. (2005) Induction of initial cardiomyocyte $\alpha$-actin - smooth muscle $\alpha$-actin - in cultured avian pregastrula epiblast: a role for Nodal and BMP antagonist. Dev. Dyn. 233; 1419-1429.

24. Nakajima, Y., Yamagishi, T., Ando, K. and Nakamura, H. (2002) Significance of bone morphogenetic protein-4 function in the initial myofibrillogenesis of chick cardiogenesis. Dev. Biol. 245; 291-303.

25. Rudy, D. E., Yatskievych, T. A., Antin, P. B. and Gregorio, C. C. (2001) Assembly of thick, thin, and titin filaments in chick precardiac explants. Dev. Dyn. 221; 61-71.

26. Sanger, J. W. and Sanger, J. M. (2001) Fishing out points that bind to titin. J. Cell Biol. 154; 21-24.

27. Schultheiss, T. M., Burch, J. B. E. and Lassar, A. B. (1997) A role for bone morphogenetic proteins in the induction of cardiac myogenesis. Genes Dev. 11; 451-462.

28. Skalli, O., Ropraz, P., Trzeciak, A., Benzonana, G., Gillessen, D. and Gabbiani, G. (1986) A monoclonal antibody against $\alpha$ smooth muscle actin: a new probe for smooth muscle differentiation. J. Cell Biol. 103; 2787-2796.

29. Sugi, Y. and Lough, J. (1992) Onset of expression and regional deposition of alpha-smooth and sarcomeric actin during avian heart development. Dev. Dyn. 193; 116-124.

30. Turnacioglu, K. K., Mittal, B., Dabiri, G. A., Sanger, J. M. and Sanger, J. W. (1997) Zeugmatin is part of the Z-band targeting region of titin. Cell Struct. Funct. 22; 73-82.

31. Yatskievych, T. A., Ladd, A. N. and Antin, P. B. (1997) Induction of cardiac myogenesis in avian pregastrula epiblast: the role of the hypoblast and activin. Development 124; 2561-2570. 